\title{
Surface expression of Cytokine Receptor-Like Factor 2 increases risk of relapse in pediatric acute lymphoblastic leukemia patients harboring IKZF1 deletions
}

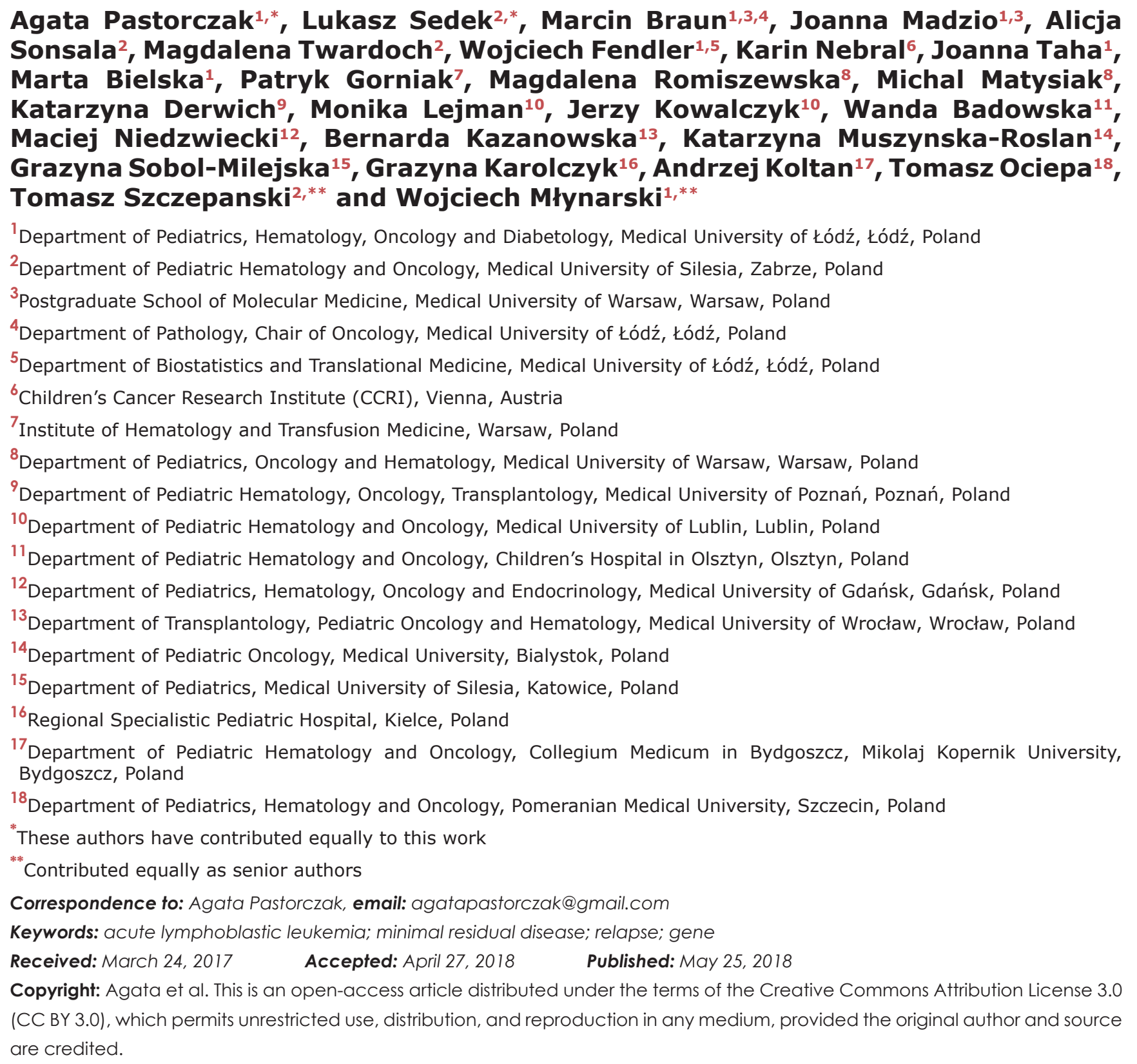

\section{ABSTRACT}

We prospectively examined whether surface expression of Cytokine ReceptorLike Factor 2 (CRLF2) on leukemic blasts is associated with survival and induction treatment response in pediatric B-cell precursor acute lymphoblastic leukemia (BCP-ALL) patients. Flow cytometric analysis of bone marrow-derived leukemia cells revealed that $7.51 \%(29 / 286)$ of 386 pediatric BCP-ALL patients were CRLF2-positive 
(CRLF2pos) at diagnosis. The median minimal residual disease (MRD) was lower in CRLF2pos than CRLF2-negative (CRLF2neg) patients on day 15 (MRD15) after induction therapy $[0.01 \%(0.001-0.42 \%)$ vs. $0.45 \%(0.05-3.50 \%) ; p=0.001]$. By contrast, the MRD15 was higher in Ikaros family Zinc Finger Protein 1 (IKZF1)-deleted BCP-ALL patients than in BCP-ALL patients without IKZF1 deletions [1.18\% $(0.06-$ $12.0 \%)$ vs $0.33 \%(0.03-2.6 \%) ; p=0.003]$. Subgroup analysis showed that MRD15

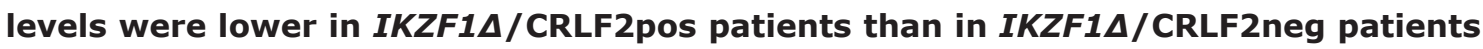
[0.1\% (0.02-5.06\%) vs. 2.9\% (0.25-12\%); $p=0.005]$. Furthermore, MRD15 levels were higher in IKZF1WT/CRLF2neg patients than in IKZF1WT/CRLF2pos patients $[0.40 \%(0.04-2.7 \%)$ vs. $0.001 \%(0.001-0.01 \%)]$. Despite the low MRD15 levels, IKZF1 $\triangle$ /CRLF2pos patients showed poorer relapse-free survival (RFS) than other patient groups $(p=0.003)$. These findings demonstrate that surface CRLF2 expression is associated with increased risk of relapse in pediatric BCP-ALL patients harboring IKZF1 deletions.

\section{INTRODUCTION}

Acute lymphoblastic leukemia (ALL) is the most common pediatric malignancy and is characterized by long-term survival rates (80-85\%), especially in developed countries [1]. In the remaining $15-20 \%$ of pediatric ALL patients, relapse is the major cause of death [2-5]. Highresolution genome-wide profiling and sequencing studies demonstrate that the risk of ALL relapse is associated with specific biological features of the leukemic cells including gene mutations, copy number variations and gene fusions. Large-scale genetic profiling of Philadelphia chromosome $(\mathrm{Ph}$ )-like ALL or $B C R$ - $A B L 1$-like ALL (Ph-positive ALL without BCR-ABL1 fusion protein) shows large number of genetic alterations in the cytokine receptor and kinasesignaling pathway genes that contribute to its aggressive phenotype and frequent disease recurrence $[6,7]$.

Nearly $47 \%$ of $B C R-A B L 1$-like ALL cases show rearrangements in the Cytokine Receptor-Like Factor 2 (CRLF2) gene [6]. CRLF2 gene encodes the thymic stromal lymphopoetin receptor (TSLPR) and is located in the pseudoautosomal region 1 (PAR1) on the Xp22.3 and Yp11.3 chromosome [8]. During lymphopoiesis and inflammation, thymic stromal lymphopoietin (TSLP) binds to the heterodimer of TSLPR and the IL7RA $\alpha$ chain $[8,9]$. $C R L F 2$ rearrangements include interstitial focal deletions juxtaposing CRLF2 with the promoter region of the G-protein purinergic receptor (P2RY8) [8, 9], or translocation of $C R L F 2$ to the immunoglobulin heavy chain locus (IGH $\alpha$ ) on 14q32.3 [6]. In both cases, CRLF2 expression is enhanced, which increases activation of the downstream JAK-STAT signaling pathway that promotes proliferation of leukemic cells $[10,11]$. In some cases, activating mutations in the CRLF2 or IL7RA genes promote CRLF2 overexpression $[8,9]$.

The prognostic relevance of genomic aberrations in the CRLF2 gene or CRLF2 mRNA overexpression in pediatric BCP-ALL is unclear because of variations in methodologies, inclusion criteria and treatment protocols used by different studies [12-16]. Bugarin et al. demonstrated that high surface CRLF2 expression correlates with increased CRFL2 transcript levels in the leukemia cells isolated from CRLF2-overexpressing BCPALL patients [17]. However, the prognostic relevance of CRLF2 protein expression in pediatric BCP-ALL patients has not been reported. Moreover, the minimal residual disease (MRD) level in BCP-ALL patients with high CRLF2 transcript levels is controversial. Therefore, in this study, we prospectively analyzed bone marrow samples from 386 pediatric BCP-ALL patients at diagnosis and at days 15 and 33 of the treatment according to ALLIC BFM 2009 protocol to determine the association of CRLF2 protein expression on the surface of leukemic cells with blast clearance in the bone marrow and treatment outcomes.

\section{RESULTS}

\section{Basic characterization of pediatric BCP-ALL patients}

Figure 1 describes the selection strategy employed in this study. Flow cytometry analysis at diagnosis showed that 29 out of 386 BCP-ALL patients (7.51\%) expressed CRLF2 (TSLPR) protein on the surface of the leukemic cells. Table 1 summarizes the clinical and biological features of all CRLF2-positive (CRLF2pos; 29/386) and CRLF2-negative (CRLF2neg; 357/386) BCP-ALL patients at diagnosis and the level of minimal residual disease at days 15 and 33 of the treatment. In comparison to CRLF2neg patients, higher number of CRLF2pos patients were associated with Down syndrome (4/29 vs. $3 / 357, \mathrm{p}=0.002)$ and $I K Z F 1$ deletions (10/29 vs. $55 / 357$, $\mathrm{p}=0.02$ ). However, both CRLF2pos and CRLF2neg BCPALL patient groups showed similar distribution of age, sex, initial WBC count, and risk group characteristics (Table 1). 


\section{CRLF2 surface expression patterns BCP-ALL patients at diagnosis}

CRLF2 expression patterns in CRLF2pos patients are shown in Table 2 and Figure 2. We observed 5 distinct patterns of CRLF2 expression in the CRLF2pos patients at diagnosis. These include [1] strong homogeneous expression of CRLF2 on leukemic blasts $(n=6$; nMFI-scores of $6-10 ; 99-100 \%$ positive); weak/dim homogeneous expression of CRLF2 ( $\mathrm{n}=6$; nMFI-scores of $2-5 ; 87.3-100 \%$ positive); [3] bimodal dim expression of CRLF2 on leukemic blasts suggesting the presence of a subclone ( $\mathrm{n}=9$; nMFI-scores of $1-2 ; 5.3-39.5 \%$ positive); [4] bimodal high expression of CRLF2 ( $\mathrm{n}=1$; nMFI-score of $6 ; 76 \%$ positive); and [5] low heterogeneous (negativeto-dim) expression of CRLF2 ( $\mathrm{n}=7$; nMFI-score of 1 ; $5.0-41.3 \%$ positive.

\section{Cytogenetic and microdeletion profiles of $\mathrm{BCP}$ - ALL patients}

Primary chromosomal abnormalities were rare in leukemic cells from CRLF2pos BCP-ALL patients based on cytogenetic analysis. Cytogenetic analysis identified
CRLF2pos patients with ETV6-RUNX1 fusion (2/29), high hyperdiploid karyotype (4/29) and MLL-ALF4 t (4; 11) gene arrangement (1/29; Table 2). These abnormalities were observed in BCP-ALL samples showing low heterogeneous (negative-to-dim) expression of CRLF2.

We screened 380 out of 386 BCP-ALL patients (98\%) for the most common microdeletions using the MLPA method. Supplementary Table 2 shows the distribution of copy number abnormalities in CRLF2pos and CRLF2neg patients. Among the 29 CRLF2pos patients, 10 showed IKZFI deletions; 5 of the 10 with IKZF1 deletions also showed P2RY8-CRLF2 fusions. $P A X 5$ and $C D K N 2 A / B$ microdeletions were observed in 8 and 9 CRLF2pos patients, respectively (Supplementary Table 1). BTG1 deletions were more prevalent in CRLF2pos (3/29) than the CRLF2neg cases (23/353). However, the statistical data was insignificant. Since concurrent $E R G$ and $I K Z F 1$ deletions are prognostically significant in ALL patients [18], we analyzed 309 ALL samples (309/386; 80\%) for the presence of $E R G$ deletions. We observed $E R G$ deletions in 14 cases $(14 / 309 ; 4.4 \%)$, of which only one patient showed high CRLF2 expression (Supplementary Table 2).

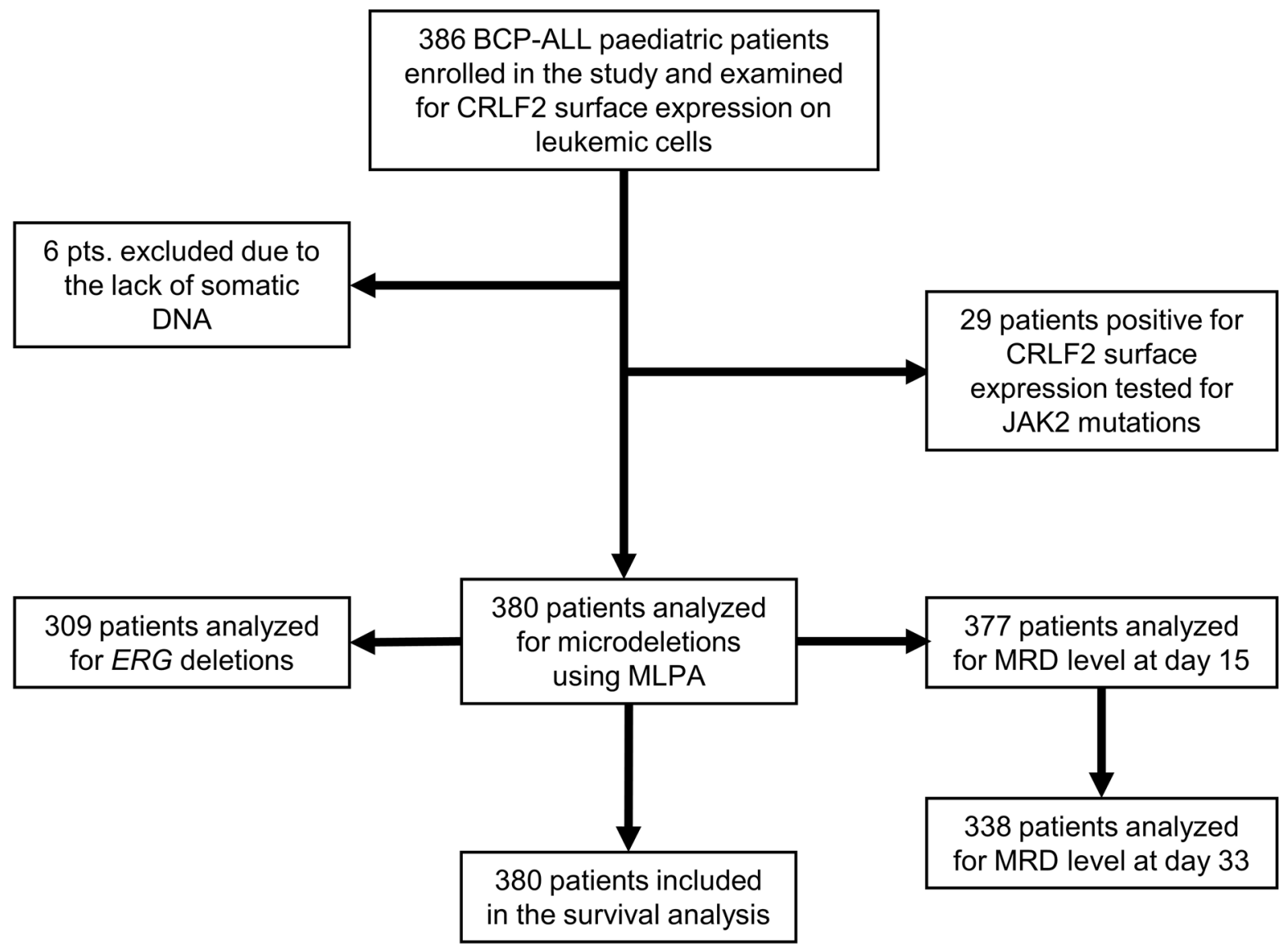

Figure 1: Flowchart shows selection strategy and classification of pediatric BCP-ALL patients at diagnosis and at days 15 and 33 of the treatment. 
Table 1: Clinical and biological characteristics of pediatric BCP-ALL patients based on CRLF2 expression status

\begin{tabular}{|c|c|c|c|}
\hline Variable & $\begin{array}{c}\begin{array}{c}\text { CRLF2neg } \\
\mathbf{n}=\mathbf{3 5 7}\end{array} \\
\end{array}$ & $\begin{array}{c}\text { CRLF2pos } \\
n=29\end{array}$ & P value \\
\hline Median age $\left(\right.$ years) ${ }^{b}$ & $4.3[2.7-7.4]$ & $4.3[3.0-7.0]$ & 0.68 \\
\hline Males $^{a}$ & $190(53)$ & $17(59)$ & 0.71 \\
\hline WBC count $\left(10^{9} / L\right)^{b}$ & $10.3[4.0-28.7]$ & $8.5[4.9-23.7]$ & 0.91 \\
\hline Poor steroid response ${ }^{a}$ & $34(10)$ & $3(10)$ & 0.77 \\
\hline Down syndrome ${ }^{a}$ & $3(2)$ & $4(36)$ & 0.002 \\
\hline Hiperdiploidy ${ }^{a}$ & $76(23)$ & $4(13)$ & 0.39 \\
\hline Hipodiploidy & $2(0.6)$ & $0(0)$ & 0.99 \\
\hline$M L L$ rearrangements ${ }^{\mathrm{a}}$ & $18(5.1)$ & $1(3.4)$ & 0.96 \\
\hline ETV6-RUNX1 fusion ${ }^{\mathrm{a}}$ & $41(15)$ & $2(6.8)$ & 0.39 \\
\hline$B C R-A B L 1$ fusion $^{a}$ & $9(2.5)$ & $0(0)$ & 0.97 \\
\hline$E R G$ deletion $^{a}$ & $13(4.5)$ & $1(3.4)$ & 0.98 \\
\hline IKZF1 deletion ${ }^{\mathrm{a}}$ & $55(16)$ & $10(35)$ & 0.02 \\
\hline Median MRD15 (\%) & $0.45[0.05-3.50]$ & $0.01[0.001-0.42]$ & 0.001 \\
\hline $\begin{array}{l}\text { Median MRD33 (\%) } \\
\text { (range) })^{b}\end{array}$ & $\leq 0.001[0.001-40.80]$ & $\leq 0.001[0.001-26.20]$ & 0.827 \\
\hline
\end{tabular}

${ }^{\text {a }}$ Shown as number of cases, with percentages in brackets.

${ }^{\mathrm{b}}$ Shown as median value with interquartile range shown in square brackets.

We detected P2RY8-CRLF2 fusion in all 13 CRLF2pos cases harboring the PAR1 deletion and 11 of these 13 cases showed dim or strong homogeneous surface expression of CRLF2. Since JAK2 point mutations are associated with CRLF2 mRNA overexpression and treatment response in ALL patients [13], we sequenced the fragment of the $J A K 2$ gene that encodes the kinase and pseudokinase domains and identified $J A K 2$ mutations in 7 CRLF2pos samples. Among these, five samples showed JAK2 mutations within the sequence encoding the pseudokinase domain (R683G) and two samples showed mutations in the kinase domain (T875A and T875N). Moreover, four samples with $J A K 2$ mutations showed P2RY8-CRLF2 fusion. These four samples showed homogeneous dim or strong expression of CRLF2. None of the 29 CRLF2pos patients showed any point mutations in the $C R L F 2$ gene.

\section{Association of CRLF2 expression and MRD status at day 15 and 33}

We prospectively analyzed the MRD status at days 15 (MRD15) and 33 (MRD33) of the treatment in 377 and 338 patients, respectively. The probability of 5-year relapse-free survival (RFS) in the study cohort based on MRD15 status is presented in Supplementary Figure 1. CRLF2pos patients showed lower MRD15 levels than CRLF2neg patients $(0.01 \%$ vs. $0.45 \%, \mathrm{p}=0.001)$.
However, the median MRD33 level was similar for both CRLF2pos and CRLF2neg patients [MRD33 CRLF2pos $\leq 0.001 \%$ (range $0.001-26.20 \%$ ); MRD33 CRLF2neg $\leq 0.001 \%$ (range $0.001-40.80 \%$ ); $\mathrm{p}=0.827$ ]. In contrast, patients with IKZFI deletion showed higher median MRD15 and MRD33 levels than patients without IKZF1 deletion [MRD15: IKZF1 $\Delta=1.18 \%(0.06-12.00 \%)$ vs. IKZF1WT $=0.33 \%(0.03-2.60 \%), \quad \mathrm{p}=0.003 ; \quad$ MRD33: IKZF $1 \Delta=0.001 \%(0.001-32.00 \%)$ vs. IKZF $1 W T=0.001 \%$ $(0.001-40.80 \%), p=0.02]$. Moreover, as shown in Figure 3 , subgroup analysis showed that MRD15 level was lowest in CRLF2pos patients without IKZF1 deletion (IKZF1WT/CRLF2pos: 0.001\% [0.001-0.01\%]), higher in CRLF2pos with IKZF1 deletion (IKZF1 $\triangle$ /CRLF2pos: $0.10 \%[0.02-5.06 \%])$ as well as CRLF2neg without IKZFI deletion (IKZF1WT/CRLF2neg: $0.40 \%$ [0.04-2.70\%]), and highest in CRLF2neg patients with IKZF1 deletion (IKZF1 $\triangle$ /CRLF2neg: $2.90 \%$ [0.25-12.0\%], p=0.0001). Multivariate analysis confirmed the mitigating effect of CRLF2 overexpression in patients with $I K Z F 1$ deletions (Supplementary Table 3). The CRLF2/IKZF1 status significantly affected the MRD15 and MRD33 levels in BCP-ALL patients after adjusting for age at diagnosis and initial WBC count. Patients with CRLF2pos/IKZF1WT genotype showed lowest MRD15 and MRD33 levels, whereas patients with CRLF2neg/IKZF1 $\Delta$ showed highest MRD15 and MRD33 levels than the other groups $(\mathrm{p}=0.003)$. CRLF2pos patients with IKZF1 deletion 
and CRLF2neg patients with IKZF1 deletion showed similar MRD15 and MRD33 levels $(p=0.95)$. MRD15 level correlated with the percentage of blasts positive for CRLF2 surface expression at diagnosis. Patients with higher percentage of CRLF2 positive blasts at diagnosis showed lower MRD15 (R=-0.43, $<<0.05$; Supplementary Figure 2).

\section{Prognostic significance of CRLF2 expression at diagnosis}

The median follow-up time for the study subjects was 4.09 (3.56-4.78) years. Univariate analysis showed that there was no correlation between CRLF2 expression status and overall survival (OS) or relapse-free survival (RFS). OS and RFS rates in CRLF2pos and CRLF2neg BCP-ALL patients were similar (OS: 88\% vs. 90\%; $\mathrm{p}=0.73$; RFS: $71 \%$ vs. $85 \%$; $=0.48)$. Overall, we noted six adverse events in CRLF2pos patients. These included five bone marrow relapses and one death due to complications in a patient with Down syndrome. Among these, three patients belonged to the intermediate risk group and the remaining three belonged to the high-risk group. Moreover, two patients that died due to relapse showed $J A K 2$ mutations, whereas, five out of six relapse patients showed IKZFI deletions. Two patients with relapse showed high MRD15 level that exceeded 10\%. Furthermore, patients with IKZFI deletions showed lower OS and RFS rates than those without IKZFI deletions (OS: $72 \%$ vs. $94 \%, \mathrm{p}=0.01$; RFS: $62 \%$ vs. $87 \%, \mathrm{p}=0.008)$. Therefore, we re-analyzed survival rates for patients with different combinations of CRLF2 and $I K Z F 1$ genetic alterations and observed that the 5-year OS rates were similar for all subgroups (Figure 4). However, IKZF1 $\triangle /$ CRLF2pos patients showed lower RFS rates than IKZ1WT/CRLF2neg patients (Figure 4). We also analyzed if $P 2 R Y 8-C R L F 2$ fusion correlated with patient survival. Our results showed no significant differences in OS and RFS between the P2RY8-CRLF2pos and P2RY8CRLF2neg groups (RFS: $84 \%$ vs. $88 \%$, $\mathrm{p}=0.42$; OS: $92 \%$ vs. $90 \%, p=0.90$; Figure 4 ).

\section{DISCUSSION}

High CRLF2 mRNA expression and IKZF1 deletions were associated with low survival rates in pediatric BCP-ALL [6]. However, prognostic relevance
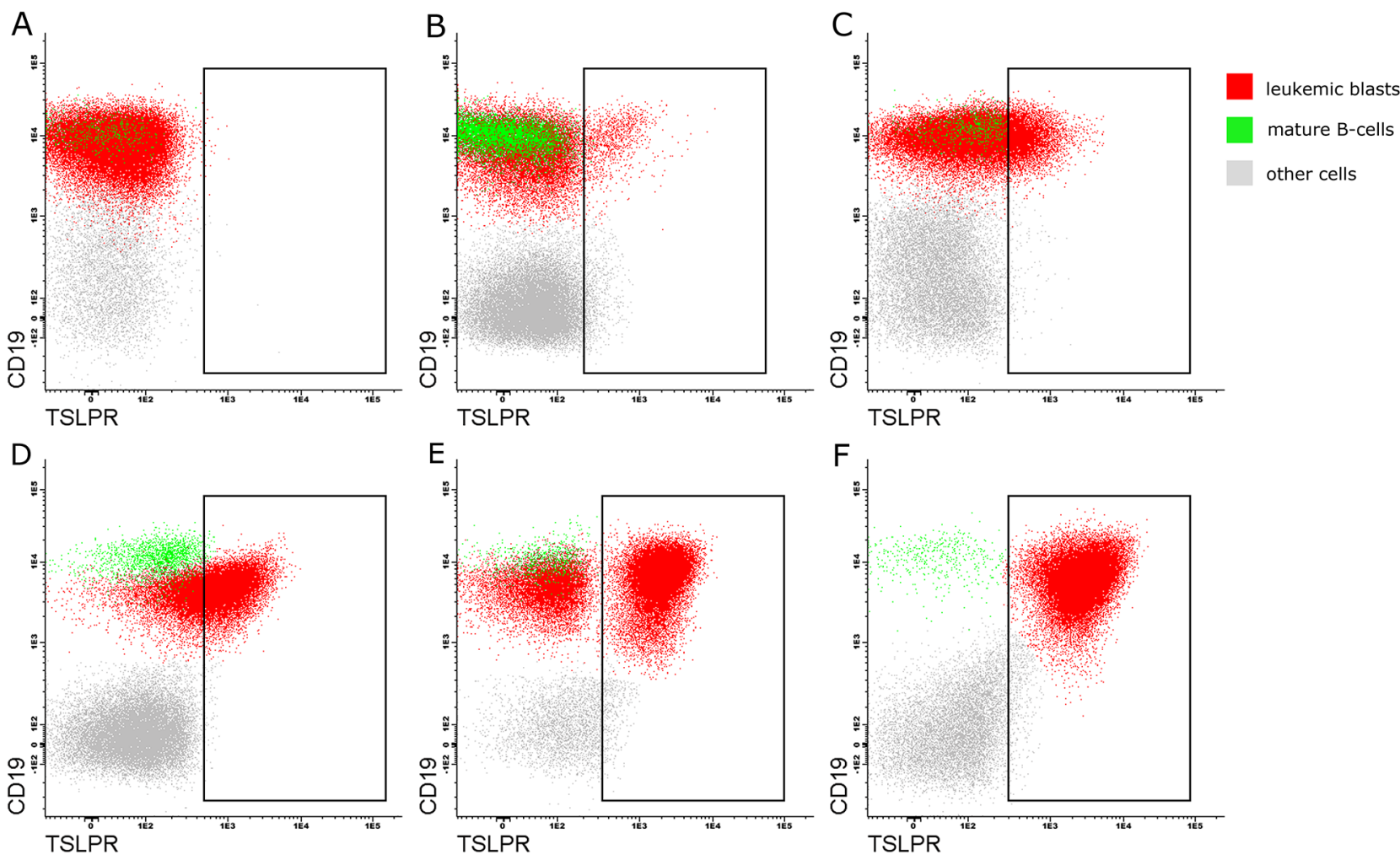

Figure 2: Flow cytometry profiles showing different CRLF2 expression patterns on leukemic blasts in the pediatric BCP-ALL patients. Representative FACS plots showing surface CRLF2 staining in (A) negative control sample and CRLF2pos patients with (B) bimodal dim pattern (low expression on the blast subclone); (C) heterogeneous low expression (negative to dim) in some blasts; (D) homogeneous dim expression on all blasts; (E) bimodal strong pattern (high expression on the blasts subclone); and (F) homogeneous strong expression on all blasts. Note: The rectangles represent blasts positive for CRLF2; red - leukemic blasts; green - mature B-cells (CRLF2-negative), gray - other cells (CRLF2-negative). 
Table 2: Detailed characteristics of CRLF2 expression and accompanying genetic aberrations on leukemic blasts and negative reference cells (mature B-cells)

\begin{tabular}{|c|c|c|c|c|c|}
\hline Sample ID & $\%$ positivity & MFI & nMFI-score & $\begin{array}{c}\text { CRLF2 expression } \\
\text { mode }\end{array}$ & Genetic aberrations \\
\hline 1330 & 36.9 & 112.1 & 1 & neg-to-dim & Hiperdiploidy \\
\hline 0959 & 27.4 & 114.8 & 1 & neg-to-dim & ETV6-RUNX1 \\
\hline 1288 & 32.9 & 117.9 & 1 & neg-to-dim & $\mathrm{t}(4 ; 11)$ \\
\hline 1584 & 5.0 & 121.9 & 1 & neg-to-dim & Hiperdiploidy \\
\hline 1020 & 41.3 & 137.7 & 1 & neg-to-dim & $I K Z F I \mathrm{del}$ \\
\hline 1704 & 6.0 & 138.7 & 1 & neg-to-dim & $I K Z F 1 \mathrm{del}, J A K 2 \mathrm{mut}$ \\
\hline 1089 & 18.4 & 163.9 & 1 & neg-to-dim & $P 2 R Y 8-C R L F 2$ \\
\hline 1322 & 9.0 & 272.41 & 1 & bimodal dim & \\
\hline 1443 & 6.1 & 295.0 & 1 & bimodal dim & $P 2 R Y 8-C R L F 2, I K Z F 1 \mathrm{del}$ \\
\hline 981 & 7.6 & 299.4 & 1 & bimodal dim & Hiperdiploidy, JAK2mut \\
\hline 1266 & 5.5 & 316.2 & 1 & bimodal dim & $I K Z F 1 \mathrm{del}, J A K 2 \mathrm{mut}$ \\
\hline 1647 & 5.3 & 404.0 & 2 & bimodal dim & ETV6-RUNX1 \\
\hline 1460 & 9.2 & 408.5 & 2 & bimodal dim & $\begin{array}{l}I K Z F I \text { del, ERGdel, } \\
\text { Hiperdiploidy }\end{array}$ \\
\hline 1571 & 6.5 & 416.2 & 2 & bimodal dim & \\
\hline 0985 & 39.5 & 562.2 & 2 & bimodal dim & \\
\hline 974 & 14.4 & 579.9 & 2 & bimodal dim & $I K Z F I \mathrm{del}$ \\
\hline 0973 & 88.2 & 491.6 & 2 & $\operatorname{dim}$ & $P 2 R Y 8-C R L F 2, I K Z F 1 \mathrm{del}$ \\
\hline 1708 & 87.3 & 672.4 & 2 & $\operatorname{dim}$ & $P 2 R Y 8-C R L F 2$ \\
\hline 1061 & 100.0 & 883.3 & 3 & $\operatorname{dim}$ & $\begin{array}{c}P 2 R Y 8-C R L F 2, I K Z F 1 \mathrm{del}, \\
J A K 2 \mathrm{mut}\end{array}$ \\
\hline 1438 & 97.8 & 916.0 & 3 & $\operatorname{dim}$ & $P 2 R Y 8-C R L F 2, J A K 2 \mathrm{mut}$ \\
\hline 1431 & 99.2 & 992.3 & 3 & $\operatorname{dim}$ & $P 2 R Y 8-C R L F 2$ \\
\hline 1497 & 98.9 & 1503.1 & 5 & $\operatorname{dim}$ & $P 2 R Y 8-C R L F 2$ \\
\hline 1522 & 76.0 & 1928.8 & 6 & bimodal strong & $\begin{array}{c}P 2 R Y 8-C R L F 2, I K Z F 1 \mathrm{del}, \\
J A K 2 \mathrm{mut}\end{array}$ \\
\hline 1048 & 100.0 & 1780.5 & 6 & strong & $P 2 R Y 8-C R L F 2$ \\
\hline 1032 & 99.4 & 862.7 & 6 & strong & $P 2 R Y 8-C R L F 2, I K Z F 1 \mathrm{del}$ \\
\hline 1459 & 100.0 & 2217.0 & 7 & strong & $C R L F 2-I G H, I K Z F 1 \mathrm{del}$ \\
\hline 0982 & 99.5 & 2519.7 & 8 & strong & $\begin{array}{c}P 2 R Y 8-C R L F 2, I K Z F 1 \mathrm{del}, \\
\text { JAK2mut }\end{array}$ \\
\hline 1604 & 100.0 & 3115.7 & 10 & strong & $P 2 R Y 8-C R L F 2$ \\
\hline 1331 & 100.0 & 3408.4 & 10 & strong & \\
\hline $\begin{array}{l}\text { negative control } \\
\text { (mature B-cells) }\end{array}$ & 0.0 & $\begin{array}{c}43.55(26.11- \\
71.69)\end{array}$ & 0 & neg & \\
\hline
\end{tabular}

Note: neg, negative; neg>mod, low expression in some blasts (heterogeneous expression from low to moderate); MFI interquartile range is reported for negative control (mature B-cells). 
of CRLF2 aberrations in pediatric ALL patients is controversial. CRLF2 overexpression activates the JAK/STAT pathway and therefore represents a potential target for JAK inhibitors in cancer therapy [10, 11]. However, in contrast to $I K Z F 1$ deletions, the prognostic value of CRLF2 aberrations and/or CRLF2 mRNA high expression is not clear. Moreover, prognostic significance of CRLF2 protein expression on leukemic cells has not been studied previously. Therefore, we investigated the clinical significance of surface CRLF2 protein expression in pediatric ALL patients at diagnosis as well as early response to the induction therapy at days 15 and 33 . We also assessed the survival outcomes in ALL patients treated according to the BFM 2009 protocol, based on surface CRLF2 expression status with or without $I K Z F 1$ deletions.

We detected surface expression of CRLF2 in $8 \%$ of BCP-ALL patients analyzed. These findings corroborate previous BCP-ALL studies (AIEOP-BFM 2000, St Jude and COG, DCOG ALL-10) that reported high CRLF2
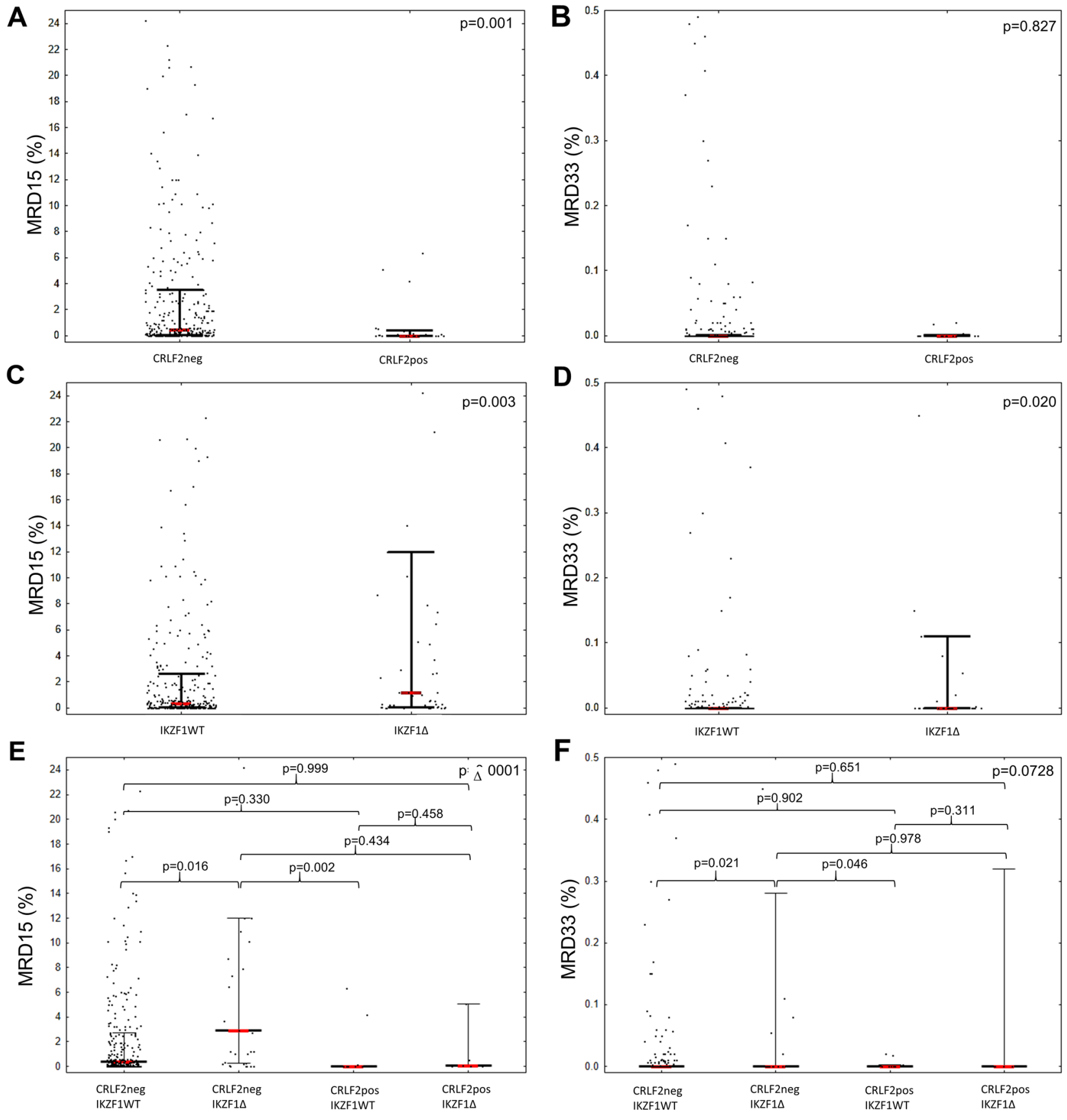

Figure 3: Comparison of MRD levels at (A, C, E) day 15 and (B, D, F) day 33 in patients with (A-B) surface CRLF2 expression, (C-D) IKZF1 deletion and (E-F) CRLF2pos patients with IKZFI deletion. 
mRNA expression in 5-7\% ALL patients [12-15]. However, this number of ALL cases with high CRLF2 mRNA expression based on the presumed cut-off values for positivity in different methodological approaches; nearly $17 \%$ of ALL cases showed high CRLF2 mRNA expression in studies that used highly sensitive qualitative

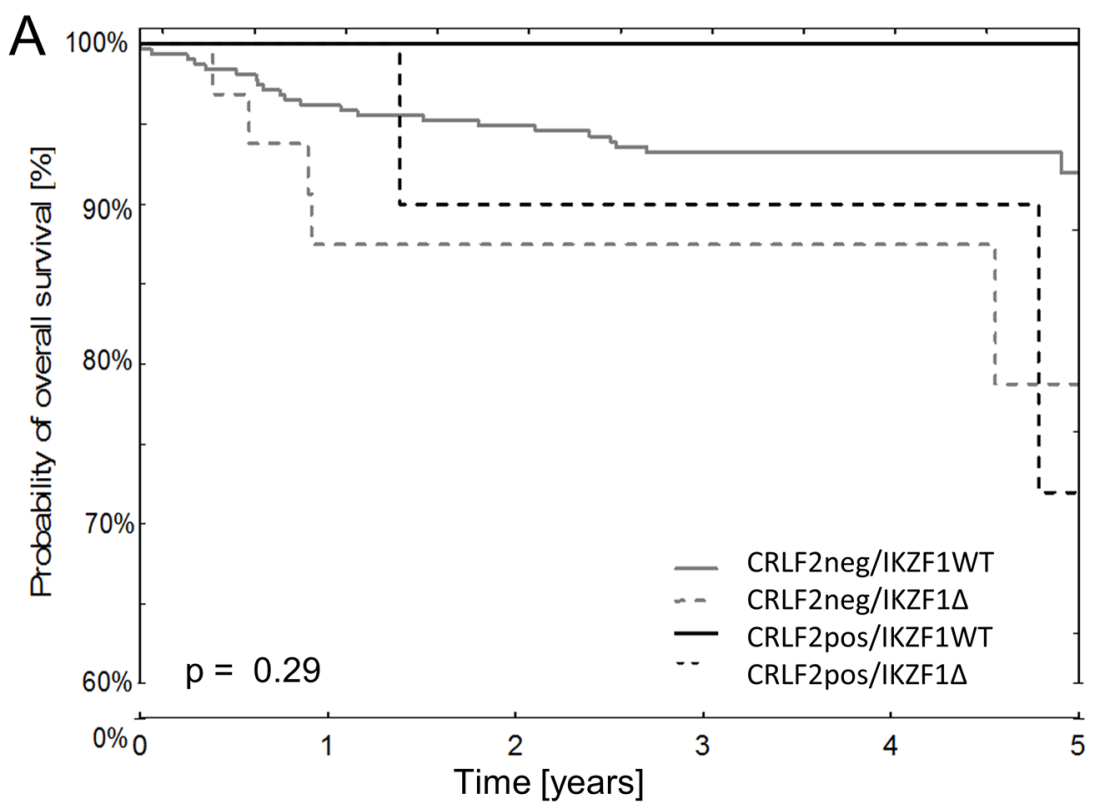

\begin{tabular}{|l|c|c|c|}
\hline & p-value & Hazard ratio & $\begin{array}{c}95 \% \text { Confidence } \\
\text { Interval }\end{array}$ \\
\hline CRLF2neg/IKZF1 & 0.140 & 2.26 & $0.76-6.68$ \\
\cline { 2 - 4 } CRLF2pos/IKZF1WT & 0.992 & 0.00 & $0.00-N A$ \\
\cline { 2 - 4 } CRLF2pos/IKZF1 & 0.446 & 2.19 & $0.29-16.43$ \\
\hline
\end{tabular}

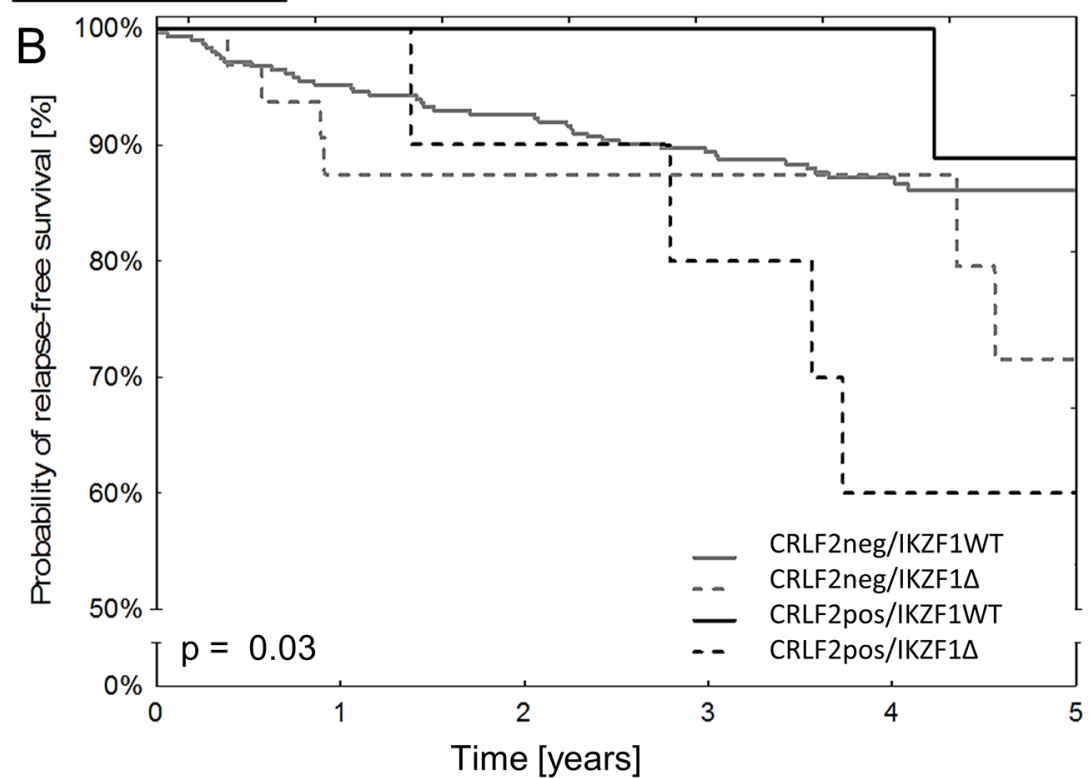

\begin{tabular}{|l|c|c|c|}
\hline & $p$-value & Hazard ratio & $\begin{array}{c}95 \% \text { Confidence } \\
\text { Interval }\end{array}$ \\
\hline CRLF2neg/IKZF1 & 0.350 & 1.50 & $0.64-3.54$ \\
\cline { 2 - 4 } CRLF2pos/IKZF1WT & 0.502 & 0.07 & $3.80-3.68$ \\
\cline { 2 - 4 } CRLF2pos/IKZF1 $\Delta$ & 0.005 & 3.72 & $1.47-9.40$ \\
\hline
\end{tabular}

Figure 4: Survival outcomes in pediatric BCP-ALL patients based on the status of CRLF2 expression and IKZF1 deletions. The Kaplan-Meier survival curves show (A) overall survival (OS) and (B) relapse-free survival (RFS) of pediatric BCP-ALL patients based on the status of surface CRLF2 protein expression and IKZF1 deletions. Also included are tables showing survival estimates based on the Cox proportional hazard regression model for different categories of patients. 
and quantitative PCR based methods [19]. Bugarin et al. reported that $\sim 10 \%$ of pediatric BCP-ALL patients exhibited surface CRLF2 expression at diagnosis by performing flow cytometry analysis using a cutoff value of $1 \%$ positivity [17]. However, cutoff values below $2 \%$ in flow cytometry analysis can result in false positives because of non-specific staining, especially in the bone marrow samples analyzed $\geq 24 \mathrm{~h}$ after isolation [20].

We observed different patterns of surface CRLF2 expression in CRLF2pos BCP-ALL patients. Among the 386 BCP-ALL patients, 12 (3.1\%) showed dim or strong homogeneous expression of CRLF2 (nMFI-scores ranging from 2 to 10$)$, whereas, $10(2.6 \%)$ exhibited bimodal dim or strong CRLF2 expression, thereby suggesting the presence of CRLF2-positive subclones. Bugarin et al. also reported CRLF2-positive subclones in 2/421 (0.5\%) patients [17]. In our study, the size of subclones ranged from 5.3 to $14.4 \%$ of blast cells in $10 / 11$ (91\%) patients with bimodal CRLF2 expression. This was consistent with previous findings, which showed that leukemic clones with CRLF2 aberrations were not predominant at diagnosis and relapse [19].

The deletion in the pseudoautosomal region 1 (PAR1 region) results in $P 2 R Y 8-C R L F 2$ fusion and represents the most common molecular event associated with CRLF2 overexpression in BCP-ALL patients [8]. Among the 29 CRLF2pos patients in our study cohort, $44 \%$ showed the incidence of $P 2 R Y 8-C R L F 2$ fusion. Furthermore, half of the P2RY8-CRLF2 fusion-positive CRLF2pos cases showed simultaneous CRLF2 protein expression. This suggested the presence of the $P 2 R Y 8-C R L F 2$ fusion in minor subclones of the leukemic cells. This hypothesis is supported by the high concordance between P2RY8$C R L F 2$ clone size and the corresponding high CRLF2 mRNA expression in BCP-ALL patients [19].

In previous reports, late relapse results in poor outcomes among the pediatric BCP-ALL patients, especially those stratified in the intermediate risk group associated with high CRLF2 mRNA expression and $C R L F 2$ rearrangements (for example, P2RY8-CRLF2 fusion) $[15,16]$. This suggests potential clinical value in evaluating surface CRLF2 expression by flow cytometry to predict treatment failure in pediatric BCP-ALL patients. In our study cohort, $P 2 R Y 8-C R L F 2$ fusion did not significantly influence survival. However, CRLF2pos patients with IKZF1 deletion were associated with increased risk of relapse.

We determined MRD15 and MRD33 levels to investigate if initial response to treatment contributed to leukemia recurrence in CRLF2pos/IKZF1 $\Delta$ patients by analyzing blast clearance in the bone marrow of CRFL2pos patients with or without $I K Z F 1$ deletions. Previous studies have shown that BCP-ALL patients with high CRLF2 expression are more sensitive to treatment as measured by MRD levels $[14,21]$. In contrast, HR-ALL cases with high CRLF2 mRNA expression from the COG cohort are associated with higher MRD levels and poor RFS at the end of induction therapy [13]. Patients harboring IKZFI deletions alone show slower blast clearance in the bone marrow during induction therapy than the patients with the wild type $I K Z F 1$ gene $[13,14]$. In our study group, CRLF2pos patients at diagnosis were associated with low MRD15 levels. Conversely, the MRD15 level was high in patients with $I K Z F 1$ deletions and low in CRLF2pos patients with $I K Z F 1$ deletions (IKZF1 $\triangle /$ CRLF2pos). This finding corroborates a previous report by Van der Veer et $a l$, which showed increased risk of relapse in high $C R L F 2$ expressing patients with $I K Z F 1$ deletions [14]. Vesely C. et al. also reported that $I K Z F 1$ deletion was the only relapse-predicting molecular indicator in $P 2 R Y 8-C R L F 2$ positive ALL cases [22]. Since we did not assess surface CRLF2 expression during follow-up studies, we could not analyze its relationship with $I K Z F 1$ deletions at the follow-up time points.

In conclusion, our study demonstrates that surface CRLF2 expression in pediatric BCP-ALL patients harboring $I K Z F 1$ deletions are associated with increased risk of relapse.

\section{MATERIALS AND METHODS}

\section{Patient characteristics}

We prospectively enrolled 386 newly diagnosed BCP-ALL children (median age: 4.3 [2.7-7.2] y) in this study. Patients were diagnosed and treated according to the ALL IC BFM 2009 protocol between August 2011 and March 2014 in the clinical centers of the Polish Pediatric Leukemia/Lymphoma Study Group: Department of Pediatrics, Hematology, Oncology and Diabetology, Medical University of Lodz, Department of Pediatric Hematology and Oncology, Medical University of Silesia, Zabrze, Department of Pediatrics, Oncology and Hematology, Medical University of Warsaw, Department of Pediatric Hematology, Oncology, Transplantology, Medical University of Poznan, Department of Pediatric Hematology and Oncology, Medical University of Lublin, Department of Pediatric Hematology and Oncology, Children's Hospital in Olsztyn, Department of Pediatrics, Hematology, Oncology and Endocrinology, Medical University of Gdansk, Department of Transplantology, Pediatric Oncology and Hematology, Medical University of Wroclaw, Department of Pediatric Oncology, Medical University, Bialystok, Department of Pediatrics, Medical University of Silesia, Katowice, Regional Specialistic Pediatric Hospital, Kielce, Department of Pediatric Hematology and Oncology, Collegium Medicum in Bydgoszcz, Mikolaj Kopernik University, Department of Pediatrics, Hematology and Oncology, Pomeranian Medical University. Patients with T cell and mature B cell ALL were not included in the study. Detailed description of the risk group assignment is presented in the online 
Supplementary Materials and Methods. The research protocol was approved by the Ethics Committee at the Medical University of Lodz and informed consent was obtained from all participants and/or their parents.

\section{Flow cytometric assessment of CRLF2 and minimal residual disease detection in BCP-ALL}

Bone marrow samples from all BCP-ALL patients were analyzed by 8-color flow cytometry protocol subscribed by the EuroFlow Consortium [23] at the one center (Department of Pediatric Hematology and Oncology, Medical University of Silesia) in Zabrze. In addition to the EuroFlow panel for BCP-ALL [24], we added an additional tube containing a combination of TSLPR-PE (clone 1D3, Biolegend, San Diego, CA, USA), CD19-PE-Cy7 (Beckman Coulter, Brea, CA, USA), CD34-PerCP-Cy5.5 (Becton Dickinson, San Diego, CA, USA) and CD45-Pacific Orange (Life Technologies, Carlsbad, USA) antibodies. The bone marrow samples collected at diagnosis of ALL as well as at days 15 and 33 of the therapy, were incubated with 1x FACS Lyse (Becton Dickinson) for erythrocyte lysis and the cells were stained and analyzed with the FACS Canto II flow cytometer (Becton Dickinson) within 1-48 h of sample collection. At least 50,000 stained cells were acquired by FACS. The reproducibility of the results was ensured by complying with the standard operating protocols developed by EuroFlow [24] and daily quality assessment of the flow cytometer with fluorescent beads (Sphero Rainbow Calibration Particles; Spherotech, Lake Forest, IL, USA) [23]. The data was analyzed using the FACS Diva 6.1 software (Becton Dickinson).

Moreover, 377 patients on day 15 and 338 patients on day 33 were subjected to flow cytometry analysis to determine the minimal residual disease (MRD) levels after induction treatment. Bone marrow samples were subjected to bulk lysis in accordance with guidelines of the EuroFlow consortium and stained with the following cocktail of monoclonal antibodies: CD22-PE, CD34-PerCP-Cy5.5, CD10-APC, CD38-APC-H7 (Becton Dickinson, San Diego, CA, USA), CD19-PE-Cy7 (Beckman Coulter, Brea, CA, USA), CD20 (Biolegend, San Diego, CA, USA) and CD45-Pacific Orange (Life Technologies, Carlsbad, USA) antibodies. In addition, cells were stained with the nucleus staining dye, Syto-16 (Life Technologies, Carlsbad, USA) to exclude dead cells and cell debris $[24,25]$. The CRLF2 (TSLPR) expression on blast cells was determined by assessing [1] the percentage of blasts positive for CRLF2 with the cutoff set at 5\%-positivity beyond the negative control level of mature B-cells, and [2] the normalized median fluorescence intensity (nMFI). The maximal MFI value for CRLF2-stained leukemic blasts was normalized by subtracting the median MFI of the negative reference population (mature B-cells) and dividing the positively stained population into 10 equal nMFI-scores, according to a protocol reported by Sedek et al. [26]. CRLF2 expression scores of 1-5 were considered negative-to-dim for low heterogeneous expression or dim for homogeneous expression. CRLF2 expression was considered as strongly homogenous for nMFI scores $\geq$ 6. In cases with bimodal CRLF2 expression with at least $5 \%$ positive blasts, the subclone was ascribed an nMFI score that corresponded to either dim or strong bimodal groups. Representative CRLF2 expression profiles are presented in Figure 2. CRLF2 antigen was not assessed during follow-up studies.

\section{Multiplex Ligation-Dependent Probe Amplification (MLPA)}

Targeted copy number screening of eight selected loci was performed by multiplex ligation-dependent probe amplification (MLPA) using both P202-B1 and P335-B2 SALSA MLPA kits (MRC-Holland, Amsterdam, The Netherlands). Detailed MLPA protocol, DNA and RNA isolation protocol, multiplex-PCR testing of $E R G$ deletions, detection of the P2RY8-CRLF2 fusion transcript, direct sequencing of $C R L F 2$ and JAK2 genes and the statistical analyses are described in the online Materials and Methods Supplementary File.

\section{Author contributions}

AP, WM, and TS designed the study; AP, MB, LS, JM, MT, AS, AT, MB and GP collected the experimental data; MR, KN, KD, ML, WB, MN, BK, EN, KMR, GSM, GK, AK and TO collected the samples and clinical data; AP, LS, WM, TS, MB, WF, JK and MM analyzed, interpreted the data, wrote and supervised the paper; WF supervised the statistical analysis. This manuscript was reviewed and approved by all the authors.

\section{ACKNOWLEDGMENTS AND FUNDING}

This work was supported by the National Centre of Research and Development (NCBiR) project (LIDER No. 031/635/1-5/13/NCBR/2014), Polpharma Scientific Foundation (grant No. 4/19/IX/1) and the National Centre of Science (grants 2011/03/B/NZ5/05906 and N N407 254440). Flow cytometry work was financially supported by ERA-NET PRIOMEDCHILD (grant 40-41800-98-027) and internal grants from the Medical University of Silesia. WM was supported by STRATEGMED3/304586/5/ NCBR/2017.

\section{CONFLICTS OF INTEREST}

The authors declare that there are no conflicts of interest. 


\section{REFERENCES}

1. Roberts KG. The biology of Philadelphia chromosome-like ALL. Best Pract Res Clin Haematol. 2017; 30:212-221.

2. Tallen G, Ratei R, Mann G, Kaspers G, Niggli F, Karachunsky A, Ebell W, Escherich G, Schrappe M, Klingebiel T, Fengler R, Henze G, von Stackelberg A. Long-term outcome in children with relapsed acute lymphoblastic leukemia after time-point and site-ofrelapse stratification and intensified short-course multidrug chemotherapy: results of trial all-REZ BFM 90. J Clin Oncol. 2010; 28:2339-2347.

3. Eckert C, von Stackelberg A, Seeger K, Groeneveld TW, Peters C, Klingebiel T, Borkhardt A, Schrappe M, Escherich G, Henze G. Minimal residual disease after induction is the strongest predictor of prognosis in intermediate risk relapsed acute lymphoblastic leukaemia - long-term results of trial ALL-REZ BFM P95/96. Eur J Cancer. 2013; 49:1346-55.

4. Roy A, Cargill A, Love S, Moorman AV, Stoneham S, Lim A, Darbyshire PJ, Lancaster D, Hann I, Eden T, Saha V. Outcome after first relapse in childhood acute lymphoblastic leukaemia - lessons from the United Kingdom R2 trial. Br J Haematol. 2005; 130:67-75.

5. Oskarsson T, Söderhäll S, Arvidson J, Forestier E, Montgomery S, Bottai M, Lausen B, Carlsen N, Hellebostad M, Lähteenmäki P, Saarinen-Pihkala UM, Jónsson ÓG, Heyman M. Relapsed childhood acute lymphoblastic leukemia in the Nordic countries: prognostic factors, treatment and outcome. Haematologica. 2016; 101:68-76.

6. Roberts KG, Mullighan CG. Genomics in acute lymphoblastic leukaemia: insights and treatment implications. Nat Rev Clin Oncol. 2015; 12:344-357.

7. Roberts KG, Li Y, Payne-Turner D, Harvey RC, Yang YL, Pei D, McCastlain K, Ding L, Lu C, Song G, Ma J, Becksfort J, Rusch M, et al. Targetable kinase-activating lesions in Ph-like acute lymphoblastic leukemia. N Engl J Med. 2014; 371:1005-15.

8. Russell LJ, Capasso M, Vater I, Akasaka T, Bernard OA, Calasanz MJ, Chandrasekaran T, Chapiro E, Gesk S, Griffiths M, Guttery DS, Haferlach C, Harder L, et al. Deregulated expression of cytokine receptor gene, CRLF2, is involved in lymphoid transformation in B-cell precursor acute lymphoblastic leukemia. Blood. 2009; 114:2688-2698

9. Yoda A, Yoda Y, Chiaretti S, Bar-Natan M, Mani K, Rodig SJ, West N, Xiao Y, Brown JR, Mitsiades C, Sattler M, Kutok JL, DeAngelo DJ, et al. Functional screening identifies CRLF2 in precursor B-cell acute lymphoblastic leukemia. Proc Natl Acad Sci USA. 2010; 107:252-257.

10. Maude SL, Tasian SK, Vincent T, Hall JW, Sheen C, Roberts KG, Seif AE, Barrett DM, Chen IM, Collins JR, Mullighan CG, Hunger SP, Harvey RC, et al. Targeting JAK1/2 and mTOR in murine xenograft models of Ph-like acute lymphoblastic leukemia. Blood. 2012; 120:3510-3518.
11. van Bodegom D, Zhong J, Kopp N, Dutta C, Kim MS, Bird L, Weigert O, Tyner J, Pandey A, Yoda A, Weinstock DM. Differences in signaling through the B-cell leukemia oncoprotein CRLF2 in response to TSLP and through mutant JAK2. Blood. 2012; 120:2853-63.

12. Attarbaschi A, Morak M, Cario G, Cazzaniga G, Ensor HM, te Kronnie T, Bradtke J, Mann G, Vendramini E, Palmi C, Schwab C, Russell LJ, Schrappe M, et al, and Associazione Italiana di Ematologia ed Oncologia Pediatrica (AIEOP)Berlin-Frankfurt-Münster (BFM) Study Group and National Cancer Research Institute (NCRI)-Children's Cancer and Leukaemia (CCLG) Study Group. Treatment outcome of CRLF2-rearranged childhood acute lymphoblastic leukaemia: a comparative analysis of the AIEOP-BFM and UK NCRI-CCLG study groups. Br J Haematol. 2012; 158:772-77.

13. Chen IM, Harvey RC, Mullighan CG, Gastier-Foster J, Wharton W, Kang H, Borowitz MJ, Camitta BM, Carroll AJ, Devidas M, Pullen DJ, Payne-Turner D, Tasian SK, et al. Outcome modeling with CRLF2, IKZF1, JAK, and minimal residual disease in pediatric acute lymphoblastic leukemia: a Children's Oncology Group study. Blood. 2012; 119:3512-22.

14. van der Veer A, Waanders E, Pieters R, Willemse ME, Van Reijmersdal SV, Russell LJ, Harrison CJ, Evans WE, van der Velden VHJ, Hoogerbrugge PM, Van Leeuwen F, Escherich G, Horstmann MA, et al. Independent prognostic value of BCR-ABL1-like signature and IKZF1 deletion, but not high CRLF2 expression, in children with B-cell precursor ALL. Blood. 2013; 122:2622-2629.

15. Cario G, Zimmermann M, Romey R, Gesk S, Vater I, Harbott J, Schrauder A, Moericke A, Izraeli S, Akasaka T, Dyer MJ, Siebert R, Schrappe M, Stanulla M. Presence of the P2RY8-CRLF2 rearrangement is associated with a poor prognosis in non-high-risk precursor B-cell acute lymphoblastic leukemia in children treated according to the ALL-BFM 2000 protocol. Blood. 2010; 115:5393-97.

16. Harvey RC, Mullighan CG, Chen IM, Wharton W, Mikhail FM, Carroll AJ, Kang H, Liu W, Dobbin KK, Smith MA, Carroll WL, Devidas M, Bowman WP, et al. Rearrangement of CRLF2 is associated with mutation of JAK kinases, alteration of IKZF1, Hispanic/Latino ethnicity, and a poor outcome in pediatric B-progenitor acute lymphoblastic leukemia. Blood. 2010; 115:5312-5321.

17. Bugarin C, Sarno J, Palmi C, Savino AM, te Kronnie G, Dworzak M, Shumich A, Buldini B, Maglia O, Sala S, Bronzini I, Bourquin JP, Mejstrikova E, et al, and I-BFM study group. Fine tuning of surface CRLF2 expression and its associated signaling profile in childhood B-cell precursor acute lymphoblastic leukemia. Haematologica. 2015; 100:e229-32.

18. Zaliova M, Zimmermannova O, Dörge P, Eckert C, Möricke A, Zimmermann M, Stuchly J, Teigler-Schlegel A, Meissner B, Koehler R, Bartram CR, Karawajew L, Rhein P, et al. ERG deletion is associated with CD2 and attenuates the negative impact of IKZF1 deletion in childhood acute lymphoblastic leukemia. Leukemia. 2015; 29:1222. 
19. Morak M, Attarbaschi A, Fischer S, Nassimbeni C, Grausenburger R, Bastelberger S, Krentz S, Cario G, Kasper D, Schmitt K, Russell LJ, Potschger U, Stanulla M, et al. Small sizes and indolent evolutionary dynamics challenge the potential role of P2RY8-CRLF2-harboring clones as main relapse-driving force in childhood ALL. Blood. 2012; 120:5134-5142.

20. Johansson U, Bloxham D, Couzens S, Jesson J, Morilla R, Erber W, Macey M, and British Committee for Standards in Haematology. Guidelines on the use of multicolour flow cytometry in the diagnosis of haematological neoplasms. $\mathrm{Br}$ J Haematol. 2014; 165:455-88.

21. Palmi C, Vendramini E, Silvestri D, Longinotti G, Frison D, Cario G, Shochat C, Stanulla M, Rossi V, Di Meglio AM, Villa T, Giarin E, Fazio G, et al. Poor prognosis for P2RY8-CRLF2 fusion but not for CRLF2 overexpression in children with intermediate risk B-cell precursor acute lymphoblastic leukemia. Leukemia. 2012; 26:2245-53.

22. Vesely C, Frech C, Eckert C, Cario G, Mecklenbräuker A, Zur Stadt U, Nebral K, Kraler F, Fischer S, Attarbaschi A, Schuster M, Bock C, Cavé H, et al. Genomic and transcriptional landscape of P2RY8-CRLF2-positive childhood acute lymphoblastic leukemia. Leukemia. 2017; 31:1491-501.

23. Kalina T, Flores-Montero J, van der Velden VH, MartinAyuso M, Böttcher S, Ritgen M, Almeida J, Lhermitte L, Asnafi V, Mendonça A, de Tute R, Cullen M,
Sedek L, et al, and EuroFlow Consortium (EU-FP6, LSHB-CT-2006-018708). EuroFlow standardization of flow cytometer instrument settings and immunophenotyping protocols. Leukemia. 2012; 26:1986-2010.

24. van Dongen JJ, Lhermitte L, Böttcher S, Almeida J, van der Velden VH, Flores-Montero J, Rawstron A, Asnafi V, Lécrevisse Q, Lucio P, Mejstrikova E, Szczepański $\mathrm{T}$, Kalina $\mathrm{T}$, et al, and EuroFlow Consortium (EUFP6, LSHB-CT-2006-018708). EuroFlow antibody panels for standardized n-dimensional flow cytometric immunophenotyping of normal, reactive and malignant leukocytes. Leukemia. 2012; 26:1908-75.

25. Theunissen P, Mejstrikova E, Sedek L, van der Sluijs-Gelling AJ, Gaipa G, Bartels M, Sobral da Costa E, Kotrová M, Novakova M, Sonneveld E, Buracchi C, Bonaccorso P, Oliveira E, et al, and EuroFlow Consortium. Standardized flow cytometry for highly sensitive MRD measurements in B-cell acute lymphoblastic leukemia. Blood. 2017; 129:347-57.

26. Sędek Ł, Bulsa J, Sonsala A, Twardoch M, Wieczorek M, Malinowska I, Derwich K, Niedźwiecki M, SobolMilejska G, Kowalczyk JR, Mazur B, Szczepański T. The immunophenotypes of blast cells in B-cell precursor acute lymphoblastic leukemia: how different are they from their normal counterparts? Cytometry B Clin Cytom. 2014; 86:329-39. 